\title{
émulations
}

\section{Regards croisés sur la migration de retour}

\author{
Audrey Lenoël, Anda David et Annalisa Maitilasso
}

Émulations - Revue de sciences sociales

2020, n 34, «Transnationaliser le retour. Vers une révision du regard sur les

migrations de retour contemporaines ».

\section{Article disponible à l'adresse suivante}

https://ojs.uclouvain.be/index.php/emulations/article/view/lenoel

\section{Pour citer cet article}

Audrey Lenoël, Anda David et Annalisa Maitilasso, « Regards croisés sur la migration de retour », Émulations, n 34, Mise en ligne le 7 octobre 2020.

DOI : 10.14428/emulations.034.01

Distribution électronique : Université catholique de Louvain (Belgique) : ojs.uclouvain.be

(c) Cet article est mis à disposition selon les termes de la Licence Creative Commons Attribution, Pas d'Utilisation Commerciale 4.0 International. http://creativecommons.org/licenses/by-nc/4.0/

Éditeur : Émulations - Revue de sciences sociales / Presses universitaires de Louvain https://ojs.uclouvain.be/index.php/emulations

ISSN électronique : 1784-5734

$\frac{\text { PUL PRESSES }}{\text { UNIVERSITAIRES }}$ 


\title{
Regards croisés sur la migration de retour
}

\author{
Audrey Lenoël ${ }^{1}$, Anda David ${ }^{2}$ \\ et Annalisa Maitilasso ${ }^{3}$
}

\begin{abstract}
[Résumé] Longtemps négligée dans les études migratoires, la migration de retour fait l'objet d'un nombre croissant d'études, d'enquêtes et de publications depuis les années 2000. Cette introduction au numéro spécial « Transnationaliser le retour » retrace le développement d'un champ de recherche spécifique sur le retour dans les différentes disciplines des sciences sociales et présente la démarche pluridisciplinaire qui a animé ce projet éditorial. Les cinq articles, l'entretien et la conclusion composant ce numéro sont ensuite présentés, puis les éléments conceptuels constitutifs du retour sont discutés à la lumière de ces contributions. Cette introduction aborde enfin les transformations conceptuelles et les enjeux contemporains de la recherche sur la migration de retour, en revenant sur le rôle des organisations (inter-) gouvernementales d'une part, et sur les conséquences socioéconomiques de la crise sanitaire liée au coronavirus d'autre part, dans la définition des agendas de recherche sur ce sujet dans les prochaines années.
\end{abstract}

Mots-clés : migration de retour, transnationalisme, pluridisciplinarité, évolution des recherches, organisations internationales, crise.

\section{Interdisciplinary perspectives on return migration}

[Abstract] Long neglected in migration studies, return migration has been the subject of an increasing number of studies, surveys and publications over the past two decades. This introduction to the special issue "Transnationalizing Return" traces the development of a specific field of research on return in the various disciplines of the social sciences, before presenting the multidisciplinary approach that has driven this editorial project. The five articles, the interview and the conclusion composing this issue are then presented, as well as the conceptual elements constituting return, which are discussed in the light of these contributions. Finally, this introduction discusses the conceptual transformations and contemporary issues in research on return migration, looking back at the role of (inter-)governmental organizations on the one hand, and the socio-economic consequences of the coronavirus-related health crisis on the other hand, in defining the research agenda on this subject in the coming years.

Keywords: return migration, transnationalism, multidisciplinarity, evolution of research, international organizations, crisis.

Qu'il soit considéré comme l'accomplissement de l'entreprise migratoire ou comme une des options à disposition des migrants, le retour est au cœur du projet de mobilité. Le fait de partir porte presque toujours en lui-même une promesse, parfois illusoire, de revenir. Désiré, planifié, fantasmé et, en même temps, constamment repoussé, ou même rejeté, le retour relève parfois du mythe. Un mythe qui perdure même quand la possibilité concrète de revenir s'éloigne de la portée des acteurs (Sinatti, 2011). Il dévoile une dimension existentielle de l'expérience migratoire, celle-ci étant confrontée à des

\footnotetext{
${ }^{1}$ Chaire Migrations et sociétés au Collège de France et Institut des Migrations (ICM).

${ }^{2}$ Agence française de développement (AFD).

${ }^{3}$ Comité espagnol du Haut-Commissariat aux réfugiés des Nations unies (ACNUR).
} 
objectifs de vie et rapportée à des enjeux identitaires cruciaux pour les migrants. La douleur engendrée dans de nombreuses familles par l'impossibilité temporaire de procéder aux rapatriements des corps de migrants décédés, liée à la fermeture des frontières lors de la crise sanitaire de la Covid-19 en 2020, manifeste la force symbolique du retour, même après la mort ${ }^{4}$.

Si la migration de retour fait aujourd'hui l'objet d'une abondante littérature, elle a certainement été le parent pauvre des études sur les migrations par le passé. Cette négligence s'explique par les représentations longtemps sous-jacentes à ce sujet, notamment les perceptions dominantes des grandes migrations historiques que sont l'exode rural et les migrations transatlantiques de l'ancien vers le nouveau monde qui furent largement pensées (à tort) comme unidirectionnelles (Gmelch, 1980 ; Sayad, 2006). À cela s'est ajoutée l'idée, particulièrement prégnante en Europe, que les migrants de retour étaient essentiellement des retraités, donc d'un intérêt socioéconomique secondaire du point de vue de l'emploi.

L'affirmation du retour comme champ de recherche spécifique dans les études migratoires a par ailleurs été freinée par les difficultés d'ordre méthodologique à appréhender précisément le phénomène d'un point de vue quantitatif. Les efforts de définition et de quantification des migrants de retour - population rare d'un point de vue statistique - étaient contraints par les limites des sources de données disponibles, qu'il s'agisse de l'absence d'indicateurs permettant de capter le phénomène dans les recensements ou les enquêtes générales ou du manque d'enquêtes spécialisées (Rallu, 2007). Ce déficit de données empiriques est allé de pair avec une insuffisante théorisation du phénomène, celui-ci apparaissant de façon plutôt périphérique dans les théories des migrations comme indicateur de succès ou d'échec du projet migratoire (Cassarino, 2004). À partir des années 1990, des recherches pionnières sur le retour furent toutefois menées en anthropologie et en sociologie : entre autres, les travaux de George Gmelch (1993) sur les expériences des migrants de retour à la Barbade, les recherches de Luis Eduardo Guarnizo (1997) sur le retour des migrants dominicains, les analyses des dynamiques circulatoires de Loretta Baldassar (2001) dans le contexte des familles italiennes en Australie ou encore les « retours contraints » des femmes maliennes observés par Catherine Quiminal (2002). À la même période, une perspective englobant aussi bien les sociétés d'origine que celles d'accueil s'ouvrait dans les études migratoires (Gardner, 1993 ; Sayad, 1999).

Depuis une vingtaine d'années, la situation a bien changé, l'étude de la migration de retour connaissant un essor sans précédent sous l'effet du développement de deux aspects des recherches dans ce domaine. D’un côté, on observe un intérêt croissant

\footnotetext{
${ }^{4}$ Cette introduction souhaitant apporter une réflexion quant à l'actualité des retours dans le contexte de la pandémie du coronavirus, nous faisons référence à plusieurs articles de presse internationale parus au printemps 2020.

Darame M. (2020), «Coronavirus : en France, les diasporas africaines ne savent plus où enterrer leurs morts », Le Monde Afrique, 13 avril 2020. En ligne, consulté le 12 juillet 2020. URL : https://www. lemonde.fr/afrique/article/2020/04/13/coronavirus-les-africains-de-france-ne-savent-plus-ou-enterrer-leurs-morts_6036472_3212.html.
} 
pour l'impact des migrations internationales sur le développement des pays d'origine. Interroger le retour en tant que facteur facilitant ou entravant les dynamiques de développement local devient en effet un enjeu central à partir des années 2000, facilité par la disponibilité croissante de données sur ce sujet (Anarfi, Jägare, 2005; Black, King, 2004). De l'autre, l'affirmation du paradigme transnational dans les études migratoires (Glick Schiller, Basch, Blanc-Szanton, 1992) a conduit à une révision critique de la notion de retour en mettant en évidence que celui-ci pouvait n'être qu'une étape d'un parcours migratoire plus complexe (Ley, Kobayashi, 2005 ; Petit, 2007).

Aujourd'hui, si les difficultés méthodologiques restent caractéristiques de ce champ de recherche, les migrations de retour font l'objet d'un nombre grandissant d'études, de programmes de recherche et d'enquêtes. Ces dernières années ont par exemple vu la réalisation d'enquêtes spécialisées adoptant des approches multisituées ou utilisant des outils de collecte biographique permettant de reconstituer la trajectoire migratoire des individus 5 . Offrant des informations riches, ces nouvelles sources de données permettent également de produire des analyses sur des pays jusqu'ici relativement méconnus. Les données de recensements, d'enquêtes en population générale et de registres sont par ailleurs de plus en plus mobilisés pour étudier le phénomène.

Dans les différentes disciplines des sciences sociales, les perspectives se sont donc multipliées pour appréhender le retour, mettant notamment en lumière les stratégies des acteurs (Quiminal, 2002), les effets des retours sur les sociétés d'origine en termes de développement (Akesson, 2011 ; Black, King, 2004), la mise en place et les effets de politiques publiques d'aide au retour volontaire (Boccagni, Lagomarsino, 2011 ; Maitilasso, 2018) ainsi que les retours forcés (David, 2017; Flahaux, 2017). Le nombre de publications dédiées à ce sujet est en constante augmentation, particulièrement depuis le tournant du XXI ${ }^{\mathrm{e}}$ siècle ${ }^{6}$, comme l’illustre le graphique ci-dessous.

\footnotetext{
${ }^{5}$ Depuis les années 2000, de plus en plus d'enquêtes spécialisées ont fait du retour leur objet principal, notamment les enquêtes MIREM conduites au Maghreb, l'enquête du programme européen TEMPER (Temporary vs Permanent migration) conduite auprès de migrants de retour en Argentine, Roumanie, Ukraine et Sénégal, l'enquête ECM2 entre l'Espagne et l'Équateur. D'autres enquêtes spécialisées sur la migration - telles que l'enquête MAFE (Migrations entre l'Afrique et l'Europe) ont également permis de mieux appréhender le phénomène.

6 Pour une vue d'ensemble de l'évolution du champ, on peut consulter George Gmelch (1980), Véronique Petit (2007), Jørgen Carling, Elin Berstad Mortensen et Jennifer Wu (2011), et plus récemment Amélie Constant (2020) qui se concentre sur les articles à forte dominante économique.
} 


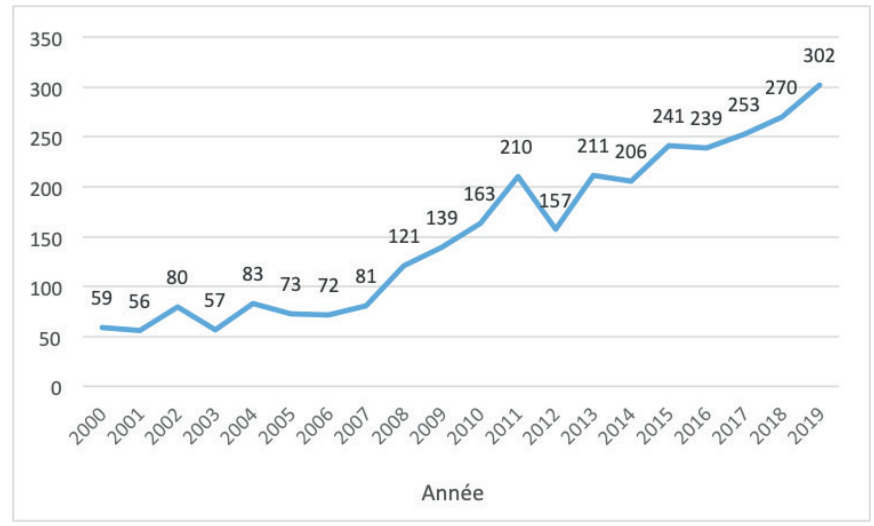

GRAPHIQUE 1 : ÉVOLUTION DU NOMBRE D'ARTICLES EN SCIENCES SOCIALES ET EN ÉCONOMIE PORTANT SUR LA MIGRATION DE RETOUR RÉFÉRENCÉS DANS LA BASE DE DONNÉES SCOPUs 2000-20197

Dans ce panorama, il est intéressant de mettre en exergue la variété des questionnements ainsi que la pluralité des prismes d'observation en fonction des disciplines. Cette diversité des perspectives témoigne de la complexité multifocale d'un objet de recherche extrêmement riche et prégnant, capable de dévoiler les mécanismes souterrains et les imaginaires qui façonnent les expériences migratoires des acteurs.

Dans cette introduction, nous commencerons par présenter notre démarche et les contributions qui composent ce numéro. Dans un second temps, nous reviendrons sur les éléments conceptuels constitutifs du retour, pour mieux les discuter à la lumière des articles de ce dossier thématique. Enfin, nous aborderons les transformations conceptuelles et les enjeux contemporains de la recherche sur la migration de retour.

\section{Une perspective pluridisciplinaire sur le retour}

Venant de champs disciplinaires différents (anthropologie, économie et sociologie), nous avons souhaité dans ce numéro croiser les regards sur ce phénomène, afin d'explorer les complémentarités de différentes approches. Si les travaux de recherche sur la migration de retour s'appuyant sur ces complémentarités entre disciplines se multiplient ${ }^{8}$, les numéros spéciaux réunissant des angles différents pour traiter ce type de migration restent encore rares. Les articles sélectionnés essayent donc de restituer la richesse d'un regard multidisciplinaire sur le retour en combinant des approches quantitatives et qualitatives et en interrogeant le sujet d'après des angles divers qui dialoguent entre eux, comme le fait ressortir Marie-Laurence Flahaux dans sa conclusion à ce numéro. Ces contributions ont été regroupées en fonction de deux axes : les trajec-

\footnotetext{
${ }^{7}$ Le graphique présenté ici correspond aux articles référencés dans Scopus pour lesquels « return migr* » apparaît dans le titre, le résumé ou les mots-clés, publiés à partir de 2000 et dans les disciplines de sciences sociales et d'économie (soit : (TITLE-ABS-KEY(return migr*) AND PUBYEAR > 1999 AND SUBJAREA(ECON OR SOCI) AND DOCTYPE(ar)).

${ }^{8}$ À titre d'exemple, les articles à dominante économique s'intéressent graduellement à des thèmes tels que le transfert des normes sociales à travers la migration de retour (Chauvet, Mercier, 2014 ; Bertoli, Marchetta, 2015), favorisant ainsi le dialogue avec des disciplines comme la sociologie et les sciences politiques.
} 
toires et expériences vécues d'une part, et le retour dans ses rapports à la communauté d'origine d'autre part.

Nous souhaitions mettre le parcours des migrants de retour au centre du premier axe sur les trajectoires et expériences vécues, avec des contributions interrogeant aussi bien la manière dont la décision de retour se prend dans un environnement de plus en plus complexe, la façon dont celui-ci est perçu par les différents acteurs, mais également les facteurs déterminant les trajectoires de retour et de réinsertion des migrants. Les articles de Marine Haddad et d’Anissa Maâ nous apportent à cet égard des éclairages intéressants et complémentaires.

Dans son article, Marine Haddad analyse les migrations de retour dans les départements français d'outre-mer (DOM) (Martinique, Guadeloupe, Guyane, la Réunion) et la manière dont elles façonnent les trajectoires familiales. Elle explore ainsi la particularité des migrations des DOM, qui sont souvent structurées par les configurations familiales, avec des familles bilocalisées dans certains cas. En appliquant aux données de l'enquête Migration, Famille et Vieillissement (MFV) - enquête menée conjointement par l'Institut national d'études démographiques (INED) et l'Institut national de la statistique et des études économiques (INSEE) entre 2009 et 2010 - des analyses de séquence portant sur la formation de la famille et les modes de cohabitation, elle met en évidence non seulement des conditions de retour différenciées entre les femmes et les hommes, mais également les trajectoires différentes qui en résultent. Ainsi, les femmes ont rencontré plus de difficultés après le retour et plus souvent, le retour n'était pour elles pas un choix, mais une contrainte. Cependant, la famille et la migration de retour s'influencent réciproquement et l'auteure approfondit son analyse en montrant que, pour les femmes, le retour retarde la mise en couple et la naissance d'enfants et fragilise les unions.

S'appuyant sur l'expérience d'une migrante ivoirienne en instance de retour à partir du Maroc, l'article d'Anissa Maâ interroge quant à lui la fabrique de la « volonté » de retour dans le cadre des programmes de « retours volontaires » mis en œuvre par des agences telles que l'Organisation internationale pour les migrations (OIM). En suivant au plus près la trajectoire d'Aya et de son nouveau-né Prince, son parcours et ses interactions avec différentes administrations, associations et l'OIM, l'auteure nous donne à voir les balbutiements d'une décision émergente de retour et le rôle des acteurs intermédiaires dans la transformation de cette dernière en une demande d'assistance auprès de l'OIM, mais également l'incertitude qui persiste dans l'issue et les modalités d'une procédure de retour volontaire. Elle fait ainsi ressortir les tensions entre pratiques des agents du retour et capacité d'agir des migrants, et met en exergue l'agentivité et les stratégies mises en place par ces derniers, même lorsque leurs marges de manœuvre semblent limitées.

Dans un second axe, nous souhaitions interroger le retour dans ses liens avec la communauté d'origine. La migration de retour fait en effet émerger des tensions entre les dimensions individuelles et collectives des stratégies de mobilité internationale 
(Baldassar, Merla, 2014). Dans cette perspective, l'article de Césarine Sambou analyse les visites effectuées par des migrants burkinabè et sénégalais installés en France à leurs familles résidant au pays d'origine. Ces retours temporaires, qui permettent de maintenir le contact avec la communauté d'origine, peuvent d'ailleurs être interprétés comme une façon de compenser la non-concrétisation d'un retour définitif. Dans ce sens, ils font émerger toute la complexité sociale du retour en Afrique de l'Ouest (également soulignée par Sylvie Bredeloup dans son entretien en fin de dossier), expérience capable de mettre en tension les inscriptions identitaires et les équilibres établis entre les migrants et la communauté locale : dans le cas concret analysé par Sambou, ceux qui reviennent en vacances se voient reprocher de manquer de solidarité en raison de leur difficulté à faire face aux attentes et aux pressions redistributives de leurs proches ; ils sont ainsi qualifiés de "toubabs », l'appellation familière réservée aux Européens de peau blanche en Afrique de l'Ouest. L'article interroge les enjeux liés à la représentation de ceux qui rentrent en illustrant une sorte de chantage symbolique mis en place par les communautés d'origine : la menace tacite d'écarter le retourné du privilège de l'appartenance et de le marquer comme autre correspond à un mécanisme de contrôle auquel les migrants ont souvent du mal à répondre (Quiminal, 2002).

La tension entre les dimensions individuelles et collectives des stratégies migratoires est également au cœur de la contribution de Samuel Lietaer, Loïc Brüning et Coumba Ndoffene Faye, dont l'article présente les observations de terrain effectuées dans trois régions du Sénégal touchées par des changements environnementaux. Les auteurs explorent les perceptions de migrants internes et de membres de leurs communautés d'origine quant aux mobilités et à l'usage des transferts de fonds, en lien avec les stratégies d'adaptation des ménages et des communautés villageoises. Si les transferts d'argent qu'elle génère peuvent être limités, la migration interne, parfois envisagée comme prélude à une mobilité internationale, permet une " double présence » physique importante des migrants. L'article montre les fortes attentes de la part des ménages d'origine envers « leurs migrants » en termes de contribution aux capacités adaptatives, mais également les variations en fonction des sites, la dimension collective de la décision de migrer et des bénéfices envisagés apparaissant en effet plus forte dans le Gandiolais et le Fouta-Toro que dans les villages du bassin arachidier. Au final, la centralité de la migration - aussi bien interne qu'internationale - dans les stratégies adaptatives explique en partie la réticence observée pour la migration de retour et une certaine prévalence de la migration de long terme.

Partant du lien complexe - et parfois ambivalent - entre retour et investissement immobilier, Anna Perraudin illustre, elle, la pluralité des stratégies mises en œuvre par les migrants équatoriens pour affronter les conséquences de la crise économique de 2008 en Espagne, notamment l'effondrement du marché du travail, l'endettement des ménages et le ralentissement de l'économie. Cet article montre comment l'acquisition de biens immobiliers, aussi bien dans le pays d'origine que dans le pays d'accueil, influence la décision de retourner en Équateur. L’auteure analyse le lien complexe et ambivalent 
entre l'achat d'une propriété et une plus grande propension au retour : dans certains cas, ces investissements contribuent à faciliter et à accélérer la décision de rentrer, tandis que dans d'autres, ils concourent à repousser ou détourner un projet de retour, en rendant possible une installation plus permanente en Espagne grâce au soutien d'une rente locative perçue dans le pays d'origine. L'originalité de cette approche repose sur la capacité à questionner la littérature qui met habituellement davantage l'accent sur le rôle ostentatoire et non productif des maisons que les migrants construisent dans le pays d'origine. L'auteure suggère que les investissements immobiliers fonctionnent comme un capital transnational, générant des ressources qui peuvent être accumulées et transférées d'un territoire à l'autre, ce qui va bien au-delà des logiques identitaire, affective ou de marqueur de mobilité sociale souvent mises en avant par la littérature.

La capacité d'agir et la réactivité des acteurs sont des aspects de la migration de retour que Sylvie Bredeloup souligne également dans l'entretien publié dans ce numéro. Parmi les différents enjeux abordés, l'anthropologue africaniste insiste sur la prédominance des retours spontanés organisés par les migrants eux-mêmes et leurs familles, en marge des programmes institutionnels d'appui au retour qui ne répondent souvent pas aux besoins et aux réalités vécues par les acteurs. Bredeloup se penche également sur la nécessité de déconstruire " les idées reçues » sur la migration de retour. Par exemple, alors qu'il est d'usage de distinguer les retours décidés librement par les individus - qualifiés alors de retours « volontaires »- de ceux qui sont contraints ou forcés, c'est-à-dire « involontaires », l'auteure note (à l'instar de Maâ) que cette dichotomie n'est pas nécessairement pertinente « sachant que la contrainte peut autant être exercée par les familles que par les États ou les organisations internationales ». À partir de ses nombreux terrains de recherche en Afrique de l'Ouest et de ses travaux sur la migration intra-africaine (entre autres, Bredeloup, Pliez, 2005 ; Bredeloup, 2020), l'auteure multiplie les exemples ethnographiques qui montrent à quel point le retour adopte des formes plurielles et des temporalités changeantes, mobilisant des logiques qui restent incompréhensibles si l'on adopte le regard simpliste et eurocentré de certaines institutions et agences.

Dans sa conclusion, Marie-Laurence Flahaux nous propose une lecture personnelle des différentes contributions de ce numéro à travers un double exercice de mise en perspective théorique, en s'appuyant sur ses propres recherches sur les retours en contexte africain. Dans un premier temps, elle fait ressortir la manière dont les concepts de " migration de retour » et de « transnationalisme » s'articulent dans les articles. Alors qu'il est généralement admis que les pratiques et les liens transnationaux influencent positivement la décision et la préparation du retour, elle met en exergue la façon dont les cas d'étude présentés par Perraudin, Sambou, ainsi que Lietaer, Brüning et Faye montrent au contraire que ces pratiques peuvent contribuer à fixer - au moins pour un temps - les migrants à destination, ou constituer des alternatives au retour. Les articles de Haddad et de Maâ lui suggèrent quant à eux que l'effet du transnationalisme sur le retour est très dépendant du profil et des trajectoires des migrants ainsi 
que du type de liens transnationaux. Dans un second temps, l'auteure fait ressortir la façon dont le retour donne à voir l'articulation entre les trajectoires de vie, familiales, matérielles, professionnelles, et migratoires des individus, mais aussi le décalage entre les aspirations des individus et les contraintes réelles auxquelles ils font face, et enfin les stratégies mises en place par les acteurs pour dépasser ces dernières.

À l'instar de Flahaux, il nous a semblé important de souligner dans cette introduction les aspects transversaux qui croisent la migration de retour, et en premier lieu le genre (Cortés, Oso, 2017). Notre démarche se veut interdisciplinaire et une lecture croisée des contributions nous montre l'apport de différentes méthodes et disciplines pour appréhender la dimension genrée du retour. Par exemple, alors que la contribution de Haddad creuse l'impact différentiel du retour dans les trajectoires des femmes et des hommes originaires des DOM à travers l'analyse de données d'une enquête sociodémographique, l'article de Maâ offre un regard socioanthropologique sur l'histoire de vie d'une migrante marquée par l'intersection de facteurs de vulnérabilité avec une claire dimension genrée, ces deux approches pouvant tout à fait être complémentaires. Si le dialogue interdisciplinaire n'est pas aisé, nous l'avons donc abordé comme un défi stimulant et nécessaire, notamment en vue de réfléchir sur des définitions du retour majoritairement partagées. Nous souhaitons donc dans un second temps revenir sur les éléments constitutifs du retour afin de mieux les discuter à la lumière des articles de ce numéro.

\section{Des retours aux contours incertains}

Pour parler de migration de retour, évoquer des retours au pluriel peut sembler plus pertinent, tant le concept est polysémique et peut désigner des situations diverses (Petit, 2007). S’il n'en existe pas de définition unique et consensuelle, on peut néanmoins distinguer les éléments caractéristiques du phénomène. Pour Bénédicte Michalon, le retour est généralement compris comme " la phase au cours de laquelle le migrant revient, pour une période plus ou moins longue, dans son espace de départ après avoir séjourné ailleurs » (2007 : 35). Cet ailleurs est généralement entendu comme un autre pays, mais peut également être dans un même pays, comme nous le verrons dans le cadre de ce numéro. On peut noter que cette définition se réfère à la mobilité d'un individu et exclut par conséquent d'autres migrations parfois qualifiées de " retours ", à savoir celles des descendants de migrants qui viendraient s'installer dans le pays d'origine de leurs aïeux ou les migrations ethniques (telles que celles des Juifs vers Israël ou des Aussiedler vers l'Allemagne après la Seconde Guerre mondiale) (Michalon, 2007). Quoi qu'il en soit, cette définition caractérise la migration de retour par une séquence de déplacements et des dimensions temporelles et spatiales, qu'il convient de discuter individuellement.

Si la dimension spatiale du retour semble assez intuitive, on peut néanmoins interroger les notions d'« espace de départ » et d'« ailleurs ». Si l'on excepte le cas des émigrations forcées, cet ailleurs est souvent compris comme un lieu de destination choisi 
par le migrant en raison des opportunités qui y seraient offertes (économiques, de réalisation personnelle, etc.). La complexification des trajectoires et l'externalisation croissante des frontières signifient cependant qu'aujourd'hui, sans doute plus qu'hier (Ahrens, Kelly, Van Liempt, 2014), nombre de retours ne s'effectuent plus depuis des pays d'émigration choisis, mais depuis des espaces considérés par le migrant comme de simples étapes vers cet ailleurs désiré. C’est le cas de la migrante ivoirienne présentée dans la contribution de Maâ, qui verra son voyage prendre fin au Maroc, alors qu'elle ne pensait passer que quelques jours dans ce pays envisagé seulement comme un espace de transit avant son départ pour l'Europe, et d'où elle prendra le chemin du retour.

L'espace de départ peut également désigner des unités diverses. Il peut par exemple s'agir du pays dont les migrants sont ressortissants ou de leur pays de naissance (Dumont, Spielvogel, 2008). Si on suppose un retour au pays de naissance, celui-ci ne se fera pas nécessairement dans la communauté d'origine du migrant. Les migrants originaires de zones rurales choisissent en effet souvent de construire et de s'installer en ville à leur retour, comme l'ont notamment montré David Lessault (2013) pour le Sénégal ou Reynald Blion (1996, mentionné par Bredeloup dans ce numéro) pour le Burkina Faso. Dans ces différents contextes, la migration internationale accélère l'urbanisation, les villes offrant aux migrants et à leurs familles de meilleures opportunités économiques et un meilleur accès aux services (Petit, 2007). Et, si l'on s'en tient à une définition large du retour, celui-ci peut également advenir à l'échelle d'un même pays, comme c'est le cas pour les migrants de retour dans les DOM après un séjour en métropole au centre de l'étude de Haddad, ou les migrants internes au Sénégal évoqués par Lietaer, Brüning et Faye.

Le concept de migration de retour se caractérise également par un critère temporel. Pour parler de migration de retour, il est généralement admis que celui-ci doit être durable, c'est-à-dire - si l'on suit les recommandations des Nations unies en matière de statistiques des migrations internationales (United Nations Statistics Division, 1998) durer au moins un an (pour les migrations de longue durée) et faire suite à un séjour à l'étranger de même durée minimale. Le retour peut également être envisagé dans le cadre de la migration de courte durée, définie par les Nations unies comme un séjour d'une durée entre trois et douze mois (Ibid.). Si ces critères sont utiles, notamment pour la démarche de définition et de quantification nécessaire à l'analyse statistique, il faut également reconnaître leur caractère arbitraire, pour distinguer par exemple un retour d'une simple visite. Pour sortir de cette impasse, certains auteurs préfèrent partir de l'interprétation que les migrants eux-mêmes proposent de leurs pratiques de retour (entre autres Sinatti, 2011). Dans ce numéro, Sambou évoque des retours qui dérogent à ce critère temporel en s'intéressant aux visites effectuées par des migrants subsahariens installés en France à leurs familles résidant au pays d'origine. Dans ce cas, l'article invite à réfléchir sur le retour temporaire en tant qu'essai général d'un retour à plus long terme. Les stratégies d'évitement mobilisées par les migrants en visite nous 
parlent d'un défi comparable à celui auquel sont confrontés les migrants de retour, sans avoir pour autant l'avantage d'une temporalité circonscrite dans le court terme. On peut également évoquer d'autres « retours » qui dérogent à ce critère temporel, à savoir les retours posthumes, notamment étudiés par Félix (2011) ou Petit (2002). Ces retours sont le symbole d'une continuité des inscriptions identitaires après le décès : comme nous l'avons déjà mentionné, les rapatriements des corps peuvent être compris comme la manifestation claire d'une volonté de retour qui se joue sur le champ des représentations et des appartenances.

Alors que l'image du migrant vieillissant retournant au pays au moment de la retraite ( le retour-retraite», comme le désigne Bredeloup) reste si prégnante en Europe, il convient également de noter que les retours peuvent s'effectuer à n'importe quelle phase de la vie, même chez les plus jeunes comme l'illustrent les travaux d'Élodie Razy (2006) sur les circulations des adolescents ouest-africains. La temporalité du retour se superpose alors à celles des différentes étapes du cycle de vie, s'influençant mutuellement. Haddad montre par exemple dans ce numéro la manière dont les trajectoires de vie et les événements clés telles que les unions et les naissances sont bouleversés par les retours pour les femmes des DOM.

Cette discussion autour du concept de retour révèle enfin une dimension supplémentaire qui doit être mobilisée pour sa définition : celle de la dialectique individu-collectivité. Comme l'illustrent les contributions de ce numéro, dans chaque retour cohabitent un projet individuel et un projet qui articule le maillage de relations familiales et communautaires autour du migrant retourné. Comme l'a notamment montré Hein de Haas (2011) dans son analyse de la formation des intentions de retour, ces relations influencent considérablement les choix personnels. Réciproquement, le retour joue un rôle important dans la création de nouveaux équilibres sociaux et économiques au sein des communautés de départ.

\section{Transformations conceptuelles et enjeux contemporains de la recherche sur la migration de retour}

Dans ce numéro, nous avons donc opté pour une définition élargie du retour qui nous paraît plus à même de révéler la richesse de cet objet d'étude. Dans ce tour d'horizon introductif, il nous semble enfin important de rappeler la prégnance des tournants conceptuels que sont l'avènement des paradigmes du transnationalisme (Glick Schiller et al., 1992) et de la mobilité (Urry, 2007) dans la compréhension du retour et, dans un second temps, d'évoquer les transformations liées au contexte contemporain qui influencent déjà et continueront d'influencer durablement l'agenda des recherches sur ce phénomène, à savoir le rôle des institutions intergouvernementales et la crise sanitaire et économique liée à la Covid-19. 


\subsection{Le retour réinterprété à la lumière des paradigmes du transnationalisme et de la mobilité}

Si, comme nous l'avons évoqué, la définition du retour formulée par les institutions et les agences internationales inclut généralement des critères de durabilité, la plupart des chercheurs jugent actuellement préférable d'éviter de penser le retour comme un mouvement nécessairement définitif. Celui-ci est aujourd'hui davantage considéré comme « un moment dans la trajectoire migratoire, et non comme une fin en soi » (Petit, 2007) ou encore " une des multiples étapes d'un mouvement continu » (Ammassari, Black, 2001). Il convient alors de recalibrer l'idée de retour en l'adaptant à des formules plus élastiques, dépourvues d'un caractère définitif (Guarnizo, 1997 ; Jeffery, Murison, 2011), capables d'inclure des situations d'entre-deux (Sinatti, 2011) ainsi que des mouvements pendulaires (Ley, Kobayashi, 2005). Ce changement du regard correspond au virage théorique représenté par les études sur le transnationalisme (Glick Schiller et al., 1992) et le paradigme de la mobilité (Urry, 2007). En d'autres termes, le concept de retour en tant que moment de conclusion de l'expérience migratoire a été remplacé par l'idée d'un projet qui évolue au fil du temps. En accord avec cette nouvelle sensibilité vis-àvis des circulations transnationales, d'autres concepts alternatifs peuvent alors être mobilisés, tels que ceux de " migration fragmentée " (fragmented migration) (Collyer, de Haas, 2012) ou de " migration continuelle » (onward migration) (Ahrens, Kelly, van Liempt, 2014).

Si ces mouvements migratoires impliquent des temporalités plus courtes, il s'agit généralement de circulations spontanées qui ont peu à voir avec la migration temporaire telle que promue par des politiques visant améliorer la gestion des flux migratoires. À la croisée du discours politique et académique, le concept de " migration temporaire » représente en réalité deux phénomènes distincts : dans le premier cas, un outil politique qui assujettit la circulation aux oscillations saisonnières de la demande de main-d'œuvre (notamment dans le secteur agricole) ; dans le second cas, une adaptation des trajectoires migratoires à des contextes en évolution. Dans le contexte actuel de durcissement des contrôles migratoires, la mobilité transnationale devient le privilège de ceux qui disposent des permis de séjour nécessaires ou qui savent comment contourner ces contrôles et ont les moyens de le faire. Enfin, il convient de noter que si le mouvement transnational, pendulaire et provisoire devient de plus en plus une forme de retour, le « retour permanent » n'a pas pour autant perdu son attrait symbolique. Même à l'ère transnationale, revenir définitivement chez soi reste une ambition pour la plupart des migrants (Sinatti, 2011).

\subsection{La recherche sur la migration de retour face à l'intérêt des organisations (inter)gouvernementales et à la crise}

Pour finir, nous souhaitions souligner deux sujets qui, parmi les transformations contemporaines du champ des recherches sur le retour, nous paraissent particulière- 
ment importants pour les années à venir : d'une part, le rôle croissant des institutions et agences (inter)gouvernementales qui, par leurs discours, leurs financements et leurs pratiques, façonnent l'approche de ces thématiques et, d'autre part, la question des retours en contexte de crise.

Pour les pays de destination, le retour effectif des migrants en situation irrégulière depuis leur territoire vers les pays d'origine tend à être une priorité politique. Les pays d'origine n'y ont en revanche souvent que peu intérêt, tant les remises migratoires représentent une manne financière indispensable pour nombre d'entre eux. En Afrique de l'Ouest, les gouvernements européens - par l'intermédiaire aussi bien de leurs agences nationales d'aide au développement que des organisations internationales qu'ils financent - tentent de juguler les flux d'émigration et de faciliter les retours par le financement d'initiatives visant à promouvoir le développement économique, la création d'emplois et des programmes de migration temporaire, sans néanmoins parvenir à s'assurer la pleine collaboration des pays d'origine, tant le coût de cette dernière en termes de politique interne est élevé (Adam et al., 2020 ; Vives, 2017). Face à ces résistances, les organisations comme l'OIM cherchent à promouvoir et à donner une image positive du retour pour inciter les migrants à revenir de manière « volontaire » et faciliter leur réintégration.

Or, comme le souligne Bredeloup dans l'entretien, de nombreux travaux ont montré « qu'il n'est pas nécessaire d'encourager les migrants pour qu'ils veuillent rentrer chez eux [et qu']ils rentrent quand ils le veulent ou le peuvent »(page 133 de ce numéro). La majorité des retours sont généralement spontanés, les incitations et prises en charge gouvernementales ou institutionnelles étant davantage l'exception que la règle. Ces programmes ont d'ailleurs souvent été des échecs relatifs si l'on en juge par le nombre de bénéficiaires, généralement bien inférieur à ce qui était escompté. Cependant, l’impact de ces programmes va bien au-delà de leurs bénéficiaires directs. Ils contribuent tout d'abord à promouvoir une idée positive des retours - incarnée dans la figure de l'entrepreneur bien décidé à développer son pays - et à en masquer la dimension contrainte à travers l'activation des registres du développement et de l'humanitaire (Bredeloup dans ce numéro). Ces programmes jouent également un rôle dans notre appréhension de l'échelle du phénomène : en effet, le manque de données chiffrées sur la migration de retour, surtout dans des contextes où la production de données migratoires n'était pas une priorité, amène souvent à décrire le phénomène à partir des chiffres de ces retours encadrés par des politiques, disponibles auprès d'organisations comme l'OIM. Cette dernière a certainement contribué à rendre ce phénomène plus visible - comme elle l'a fait pour d'autres phénomènes comme les morts aux frontières (Heller, Pécoud, 2019) et, ce faisant, à imposer l'image du migrant de retour "super entrepreneur » (Naudé, Siegel, Marchand, 2017) qui ne correspond cependant pas à la majorité des profils de retournés. Lorsque des enquêtes spécialisées sur la migration de retour enregistrent le fait d'avoir été aidé par un programme, on se rend alors compte de la faible proportion de ces cas par rapport à l'ensemble des migrants de retour : dans l'enquête TEMPER 
Sénégal, moins de $7 \%$ des 600 migrants de retour de France ou d'Espagne interrogés avaient bénéficié d'un tel programme, tandis que dans l'enquête ECM2, seuls $26 \%$ des migrants équatoriens de retour d’Espagne ayant cherché un appui avaient bénéficié d'une telle aide. Des analyses détaillées de la diversité des retours sont donc cruciales.

Pour finir, il nous paraît important de souligner l'importance de la question du retour en temps de crise dans l'agenda des futures recherches dans le domaine. Cette question est évoquée dans ce numéro, particulièrement par Lietaer, Brüning et Faye dans le contexte de crise environnementale au Sénégal (dégradation des terres, pluviométrie imprévisible, et augmentation des températures), par Perraudin concernant les retours en temps de crise économique en Espagne et par Bredeloup, qui évoque les retours de Burkinabé de Côte d'Ivoire face à la crise politique de 2002. Or, cette question de l'implication des crises pour les retours sera incontournable dans les années à venir, compte tenu de la crise économique et sanitaire mondiale liée au coronavirus qui débute en 2020. Si les effets à long terme sur les dynamiques migratoires sont encore incertains et que leur mesure nécessitera des données non encore disponibles, plusieurs médias et organisations ont rapporté des retours massifs de migrants qui avaient parfois mis des années pour atteindre l'Europe ou les États-Unis au prix de terribles sacrifices humains et financiers ${ }^{9}$. Des filières de retour clandestines se seraient même mises en place, notamment entre l'Espagne et le Maroc, proposant des trajets au prix fort pour des migrants en situation irrégulière se retrouvant en grande difficulté suite à l'entrée en vigueur de l'état d'urgence sanitaire ${ }^{10}$.

Beaucoup d'autres migrants seront restés à destination, par choix ou par nécessité, et risquent alors une précarisation accrue de leur situation, souvent déjà difficile, notamment dans les pays du sud de l'Europe. Les migrants pouvant retourner dans leur pays d'origine, dans un autre pays dans lequel ils ont déjà séjourné ou qui sont en mesure de migrer vers un pays tiers seront ceux qui auront la capacité de supporter les coûts de cette nouvelle migration, donc ceux qui ont été relativement épargnés par les impacts négatifs de la crise actuelle. Sa relative soudaineté, son échelle globale, mais également la perspective de l'augmentation de la fréquence de ce type d'événements, peuvent également façonner la manière dont le retour peut être envisagé et les stratégies que les migrants mettront en place face à l'incertitude. Ces dix dernières années, la crise économique secouant l'Europe avait déjà transformé la manière dont le retour était envisagé aussi bien par les migrants eux-mêmes que par les institutions publiques des pays d’arrivée et de départ. Si le retour était auparavant envisagé soit comme un moment

\footnotetext{
9 Semple K. (2020), «As World Comes to Halt Amid Pandemic, So Do Migrants », New York Times, 4 mai 2020. En ligne, consulté le 12 juillet 2020. URL : https://www.nytimes.com/2020/05/04/world/americas/coronavirus-migrants.html.

MandRaud I. (2020), « Coronavirus : I'exode mondial avant le confinement », Le Monde, 17 avril 2020. En ligne, consulté le 12 juillet 2020. URL : https://www.lemonde.fr/international/article/2020/04/17/ covid-19-I-exode-mondial-avant-le-confinement_6036919_3210.html.

${ }^{10}$ Martin M. (2020), « Más de 5.000 euros por escapar de España en patera », El Pais, 24 avril 2020. En ligne: consulté le 12 juillet 2020. URL : https://elpais.com/espana/2020-04-23/mas-de-5000-euros-por-escapar-de-espana-en-patera.html.
} 
lointain correspondant à la fin de la vie active, soit comme l'aboutissement de l'aventure migratoire, il est devenu pour beaucoup de migrants démunis une stratégie de sortie, une réalité imposée ou encore un horizon inatteignable. La précarité sociale et l'insécurité juridique caractéristiques de la vie de nombre d'entre eux affectent directement leurs perspectives, possibilités et représentations d'une " réinstallation durable » dans le contexte d'origine. Cette tendance pourrait donc s'accroître dans les années à venir. Un nouveau pan des recherches sur le retour va donc s'ouvrir. Ces études devraient s'appuyer sur l'important corpus de recherches de ces dernières décennies et donner toute sa place à l'interdisciplinarité.

\section{Bibliographie}

Adam I., Trauner F., Jegen L., Roos C. (2020), « West African Interests in (EU) Migration Policy. Balancing Domestic Priorities with External Incentives », Journal of Ethnic and Migration Studies, p. 1-18.

Ahrens J., Kelly M., Van Liempt I. (2014), «Free Movement? The Onward Migration of EU Citizens Born in Somalia, Iran, and Nigeria », Population, Space and Place, vol. 22, $\mathrm{n}^{\circ} 1, \mathrm{p} .84-98$.

Akesson L. (2011), " Making Migrants Responsible for Development: Cape Verdean Returnees and Northern Migration Policies », Afrika Spectrum, vol. 41, n 1, p. 61-83.

Ammassari S., Black R. (2001), " Harnessing the Potential of Migration and Return to Promote Development: Applying Concepts to West Africa », Sussex Migration Working Papers, Sussex University.

ANARFI J. K., JÄGARE S. (2005), Toward the Sustainable Return of West African Transnational Migrants: What Are the Options?, paper presented at the « New Frontiers of Social Policy » conference, Arusha, Tanzania, 12-15 December 2005.

Baldassar L. (2001), Visits Home: Migration Experiences Between Italy and Australia, Melbourne, Melbourne University Press.

Baldassar L., Merla L. (dir.) (2014), Transnational Families, Migration and the Circulation of Care: understanding mobility and absence in family life, New York, Routledge («Transnationalism Series »).

Bertoli S., Marchetta F. (2015), « Bringing it all back home-return migration and fertility choices », World Development, vol. 65, p. 27-40.

BLACK R., KING R. (2004), « Editorial introduction: migration, return and development in West Africa », Population, Space and Place, vol. 10, n² 2. p. 75-83.

Blion R. (1996), « Migrants internationaux et de retour au Burkina Faso, acteurs et témoins d'une circulation migratoire multiforme ", in R. OTAYEK, F.M. SAWAdogo, J.-P. Guingane (dir.), Le Burkina Faso entre révolution et démocratie (1983-1993), Paris, Karthala, p. 133-155. 
Boccagni P., Lagomarsino F. (2011), " Migration and the global crisis: new prospects for return? The case of Ecuadorians in Europe », Bulletin of Latin American Research, vol. 30, n 3, p. 282-297.

BREDELoup S. (2020), « La Libye, une terre d'écueil pour des migrants burkinabè ? ", in S. Bredeloup, A. Degorce, A. Palé (dir.), Se chercher en migration. Expériences burkinabè, Dakar, L'Harmattan (« Mobilités africaines »), p. 165-190.

Bredeloup S., Pliez O. (2005), « Migrations entre les deux rives du Sahara », Autrepart revue de sciences sociales au Sud, vol. 36, n 4, p. 3-20.

Carling J., Mortensen E. B., Wu J. (2011), A systematic bibliography on return migration, PRIO Paper, Oslo, Peace Research Institute Oslo.

CASSARINo J.-P. (2004), « Theorising Return Migration: the Conceptual Approach to Return Migrants Revisited », International Journal on Multicultural Societies, vol. 6, $\mathrm{n}^{\circ}$ 2, p. 253-279.

Chauvet L., Mercier M. (2014), « Do return migrants transfer political norms to their origin country? Evidence from Mali », Journal of Comparative Economics, vol. 42, n 3 , p. 630-651.

Collyer M., De HaAs H. (2012), « Developing dynamic categorisations of transit migration », Population, Space and Place, vol. 18, n 4, p. 468-481.

Constant A. (2020), « Time-Space Dynamics of Return and Circular Migration: Theories and Evidence ", GLO Discussion Paper, $\mathrm{n}^{\circ}$ 445, Essen, Global Labor Organization (GLO). En ligne. URL : https://www.econstor.eu/bitstream/10419/210984/1/GLODP-0446.pdf.

CoRTés A., Oso L. (2017), « Avecillas y pájaros en vuelo transnacional: Retorno, género y estrategias de movilidad e inmovilidad entre Ecuador y España », RES. Revista Española de Sociología, vol. 26, n 3, p. 359-372.

David A. M. (2017), « Back to Square One: Socioeconomic Integration of Deported Migrants ». International Migration Review, vol. 51, n 1, p. 127-154.

DE HAAS H. (2011), « The effects of integration and transnational ties on international return migration intentions », Demographic Research, vol. 25, p. 755-782.

Dumont J.-C., Spielvogel G. (2008), " Les migrations de retour : un nouveau regard », Perspectives des migrations internationales, SOPEMI 2008, Paris, OECD, p. 181-246.

Felix A. (2011), " Posthumous transnationalism: Postmortem Repatriation from the United States to Mexico », Latin American Research Review, vol. 46, n³, p. 157-179.

Flahaux M.-L. (2017), "The Role of Migration Policy Changes in Europe for Return Migration to Senegal », International Migration Review, vol. 51, n 4, p. 868-892.

GARDNER K. (1993), « Desh-Bidesh: Sylheti images of home and away », Man, new series, vol. 28, no 1, p. 1-15. 
Glick Schiller N., Basch L., Blanc-Szanton C. (dir.) (1992), Toward a Transnational Perspective on Migration, New York, New York Academy of Sciences.

Gmelch G. (1980), « Return Migration », Annual Review of Anthropology, vol. 9, n 1, p. 135159.

Gmelch G. (1993), Double Passage: The Lives of Caribbean Migrants Abroad and Back Home, Ann Arbor, University of Michigan Press.

Guarnizo L. E. (1997), «The Emergence of a Transnational Social Formation and the Mirage of Return Migration among Dominican Transmigrants », Identity, vol. 4, $\mathrm{n}^{\circ}$ 2, p. 281-322.

Heller C., PÉcoud A. (2019), « Counting Migrants’ Deaths at the Border: From Civil Society Counterstatistics to (Inter)Governmental Recuperation ", American Behavioral Scientist, vol. 64, $\mathrm{n}^{\circ}$ 4, p. 480-500.

Jeffery L., Murison J. (2011), " The Temporal, Social, Spatial, and Legal Dimensions of Return and Onward Migration », Population, Space and Place, vol. 17, n² 2, p. 131-139.

Lessault D. (2013), " Des espaces parcourus aux lieux investis. Enquête auprès des Sénégalais installés en Europe », Autrepart, vol. 67-68, n 4-5, p. 213-232.

Ley D., Kobayashi A. (2005), « Back to Hong Kong: return migration or transnational sojourn?», Global networks, vol. 5, n² 2, p. 111-127.

Maitilasso A. (2018), " La migración maliense en España: retornos "no asistidos” y movilidad transnacional », in J. Arango, R. Mahía, D. Moya Malapeira, E. SánchezMontijano (dir.), Anuario CIDOB de la Inmigración 2018, p. 308-328.

Michalon B. (2007), « Les retours en migration : une notion polysémique, des formes migratoires multiples », in V. PETIT (dir.), Migrations internationales de retour et pays d'origine, Paris, Éditions du CEPED, p. 27-45.

Naudé W., Siegel M., Marchand K. (2017), « Migration, entrepreneurship and development: critical questions », IZA Journal of Migration, vol. 6, n 5, p. 1-16.

Petit A. (2002), "L'ultime retour des gens du fleuve Sénégal », Hommes et Migrations, $\mathrm{n}^{\circ} 1236, \mathrm{p} .44-52$.

Petrt V. (2007), « Présentation », in V. Petrt (dir.), Migrations internationales de retour et pays d'origine, Paris, Éditions du CEPED, p. 9-26.

Quiminal C. (2002), "Retours contraints, retours construits des émigrés maliens », Hommes et Migrations, $\mathrm{n}^{\circ}$ 1236, p. 35-43.

RALLU J.-L. (2007), «L'étude des migrations de retour: données de recensement, d'enquête et de fichiers », in V. PetiT (dir.), Migrations internationales de retour et pays d'origine, Paris, Éditions du CEPED, p 47-56.

RAZY É. (2006), « De quelques “retours soninké” aux différents âges de la vie. Circulations entre la France et le Mali », Journal des anthropologues, n 106-107, p. 337-354. 
SAYAD A. (1999), La double absence. Des illusions de l'émigré aux souffrances de l’immigré, Paris, Seuil.

SAYAD A. (2006 [1998]), "Le retour élément constitutif de la condition de l'immigré », in A. SAYAD, L'immigration ou Les paradoxes de l'altérité. Tome 1, L'illusion du provisoire, Paris, Éditions Raisons d'agir ("Cours et travaux »), p. 131-192.

SinAtTi G. (2011), « "Mobile Transmigrants” or “Unsettled Returnees”? Myth of Return and Permanent Resettlement among Senegalese Migrants », Population, Space and Place, vol. 17, n² 2, p. 153-166.

United Nations Statistics Division (1998), Recommendations on Statistics of International Migration, Revision 1, New York, United Nations.

URRY J. (2007), Mobilities, Cambridge, Polity Press.

VIVES L. (2017), « The European Union-West African Sea Border: Anti-Immigration Strategies and Territoriality ", European Urban and Regional Studies, vol. 24, $\mathrm{n}^{\circ} 2$, p. 209-224. 